JOURNAL OF SYNCHROTRON RADIATION

ISSN 1600-5775

Received 1 December 2015

Accepted 18 May 2016

Edited by G. Grübel, HASYLAB at DESY, Germany

Keywords: USAXS; XPCS.

\section{Multi-speckle X-ray photon correlation spectroscopy in the ultra-small-angle $X$-ray scattering range}

\author{
Johannes Möller, Yuriy Chushkin, Sylvain Prevost and Theyencheri Narayanan*
}

ESRF - The European Synchrotron, 38043 Grenoble, France. *Correspondence e-mail: narayan@esrf.eu

Multi-speckle X-ray photon correlation spectroscopy (XPCS) measurements in the ultra-small-angle range are performed using a long pinhole collimation instrument in combination with two-dimensional photon-counting and highsensitivity imaging detectors. The feasibility of the presented setup to measure dynamics on different time and length scales pertinent to colloidal systems is shown. This setup offers new research opportunities, such as for example in the investigation of non-equilibrium dynamics in optically opaque, complex systems over length scales from tens of nanometres to several micrometres. In addition, due to the short duration of the X-ray exposure involved in the ultra-small-angle range, possible radiation-induced effects are alleviated. Furthermore, the performance of two different detectors, a photon-counting Pilatus $300 \mathrm{~K}$ and an integrating FReLoN CCD, are compared, and their applicability for accurate XPCS measurements is demonstrated.

\section{Introduction}

For nearly half a century, complex dynamics and relaxations in various condensed matter systems have been studied by using coherent visible-light scattering techniques, most prominently dynamic light scattering (DLS) (Berne \& Pecora, 2000). In this method, the dynamics within the material are manifested by the fluctuations in the coherent scattering intensity. The same principle also holds when the dynamics are probed by using a coherent X-ray beam. This technique is known as X-ray photon correlation spectroscopy (XPCS) (Mainville et al., 1997; Grübel et al., 2008; Sutton, 2008). With the increasing brilliance of synchrotron radiation sources over the last decades, numerous applications of coherent X-ray scattering methods have emerged. Among them, XPCS has been well established as a technique to probe slow dynamics in complex systems (Grübel et al., 2008; Sutton, 2008; Madsen et al., 2010).

One main assumption in the treatment of DLS and XPCS data is that every detected photon has only been scattered once by the sample, i.e. there is no multiple scattering. In the case of light scattering however, especially when used for studying concentrated systems with sizes up to several micrometres or with high refractive index contrasts, multiple scattering becomes highly probable (Segre et al., 1995a; Moussaïd \& Pusey, 1999). This needs further technical development in order to either suppress the multiple scattering as in cross-correlation methods (Segre et al., 1995b; Aberle et al., 1998; Block \& Scheffold, 2010) or to restrict the analysis to the strongly multiple-scattering regime by diffusing wave spectroscopy (Pine et al., 1988). The conventional DLS is therefore often limited to low concentrations and not readily suitable for samples which are optically opaque or highly 
absorbing. This limitation can be overcome by the use of hard X-rays, which have a much smaller scattering probability, making XPCS a valuable technique for optically opaque systems.

However, the information in the scattered intensity on a certain length scale relates to the scattering at a certain angle, given by the scattering vector of magnitude $q$, which has units of reciprocal length. Here, with $\theta$ being the scattering angle and $\lambda$ the wavelength of the incoming photons,

$$
q=4 \pi \sin (\theta / 2) / \lambda
$$

Therefore, scattering from the same length scale occurs at much smaller angles when the photon energy is increased from visible light to X-rays. In order to access length scales in the micrometre range, scattering vectors down to $q=10^{-3} \mathrm{~nm}^{-1}$ are required. This is usually difficult to reach experimentally for typical X-ray wavelengths $(\lambda \simeq 1 \AA$ ).

A standard experimental setup for XPCS, or coherent scattering in general, at a modern synchrotron beamline consists of a small-angle X-ray scattering (SAXS) pinhole camera in combination with a high-resolution two-dimensional detector (Abernathy et al., 1998; Sandy et al., 1999; Llopart et al., 2002; Westermeier et al., 2009; Shinohara et al., 2010a; Hoshino et al., 2012). Since the synchrotron is only a partially coherent source, a high degree of spatial filtering is needed to obtain a transverse coherence length of several tens of micrometres (Abernathy et al., 1998; Sandy et al., 1999; Alaimo et al., 2009). Different constraints determine the smallest accessible $q$ value in such a setup, like the maximum sample-to-detector distance, natural divergence of the X-ray beam, pixel sizes and intensity dynamic range of the available detectors, the limited numbers of pixels near to the direct beam and the size of the beamstop itself, which has to be used in order to protect the detector from the direct beam. Of course, these limitations hold true for dynamic as well as static small-angle scattering experiments. Therefore, the smallest reported $q$ values for pinhole geometry SAXS setups are of the order of $q=10^{-2} \mathrm{~nm}^{-1}$.

This issue has been addressed in the past with the construction of dedicated Bonse-Hart setups, which have successfully been employed for ultra-small-angle X-ray scattering (USAXS) experiments (Sztucki et al., 2006; Sztucki \& Narayanan, 2007; Zhang et al., 2011). A Bonse-Hart doublecrystal setup at an undulator source offers the high angular resolution needed for reaching the USAXS range as well as the coherent flux to perform XPCS experiments in this geometry (Sztucki et al., 2006; Zhang et al., 2011, 2012). However, this kind of setup has certain drawbacks since it involves a sequential acquisition at each scattering vector by a point detector. This can be a problem when investigating anisotropic and radiation-sensitive or non-equilibrium samples, which is often the case for typical soft matter systems. In contrast, with a two-dimensional detector, data over a wide range of scattering vectors and the full azimuthal range are simultaneously acquired. In addition, a direction-dependent analysis (Fluerasu et al., 2010; Burghardt et al., 2012) can be made more easily by using a two-dimensional detector. It is worth mentioning that static scattering information as well as $q$-dependent relaxation times for even smaller scattering vectors $\left(q<10^{-3} \mathrm{~nm}^{-1}\right)$ can, in principle, be obtained by analyzing the near-field speckle patterns (Cerbino et al., 2008). This analysis is, however, often complicated due to the complex spatial response function of the detector (Alaimo et al., 2009; Lu et al., 2011).

In this paper, we demonstrate XPCS measurements in the USAXS range using a pinhole geometry, which became available at the recently upgraded beamline ID02 at the ESRF (Van Vaerenbergh et al., 2015). The new focusing scheme and the new $34 \mathrm{~m}$-long flight tube equipped with different twodimensional detectors allow access to scattering angles corresponding to length scales of several micrometres. This setup offers not only the unique opportunity to perform timeresolved USAXS but also USAXS-XPCS experiments using two-dimensional detectors, which is the subject of this paper.

\section{X-ray photon correlation spectroscopy}

\subsection{Coherence and contrast}

XPCS probes the dynamic properties of matter through the temporal correlation of the scattering intensity. The homodyne intensity-intensity time autocorrelation function is defined as

$$
g_{2}\left(\mathbf{q}, t_{1}, t_{2}\right)=\frac{\left\langle I\left(\mathbf{q}, t_{1}\right) I\left(\mathbf{q}, t_{2}\right)\right\rangle}{\left\langle I\left(\mathbf{q}, t_{1}\right)\right\rangle\left\langle I\left(\mathbf{q}, t_{2}\right)\right\rangle},
$$

with $I(\mathbf{q}, t)$ being the intensity measured at wavevector $\mathbf{q}$ at time $t$ and $\langle\ldots\rangle$ the pixel average (Berne \& Pecora, 2000). With this so-called two-time correlation function, non-equilibrium and non-stationary dynamics can be investigated (Brown et al., 1997; Fluerasu et al., 2007; Shinohara et al., $2010 b$ ). For limited time intervals, however, when the overall measurement time is much smaller than typical aging times in the sample or in the case of a stationary system (e.g. Brownian motion), the two-time correlation function can be described for a (quasi-)stationary state as (Berne \& Pecora, 2000)

$$
g_{2}(\mathbf{q}, t)=\frac{\langle I(\mathbf{q}, \tau) I(\mathbf{q}, \tau+t)\rangle}{\langle I(\mathbf{q})\rangle^{2}} .
$$

Here, $\langle\ldots\rangle$ denotes the time averaging. In the limit of large times $t \rightarrow \infty$, the value of $g_{2}(q, t)$ approaches unity, as the scattering signals over large time scales are completely uncorrelated. For short delay times $t \rightarrow 0$, however, it tends to $1+\beta$, with $\beta$ being the so-called contrast, which is strongly connected to the coherence properties of the radiation, and $\beta \simeq 1$ for a single coherence volume.

With coherent illumination, the observed scattering pattern displays a typical speckle pattern, obeying negative exponential intensity statistics. The resulting probability distribution of the scattered intensity can then be written as (Goodman, 1985) 


$$
P(I)=\frac{\exp (-I /\langle I\rangle)}{\langle I\rangle} .
$$

The opposite would be the case when the scattering volume is much larger compared with the coherence volume, so that $\beta \cong$ 0 . In this case, the intensity distribution follows Gaussian statistics.

At modern synchrotron radiation beamlines, a partially coherent beam of X-rays with a high flux is produced, which can be spatially filtered for coherent scattering experiments by using an aperture smaller than the coherence area of the beam (Jakeman et al., 1976; Abernathy et al., 1998; Sandy et al., 1999). However, further experimental constraints alter the contrast of the XPCS experiment. In a typical experiment, with highly coherent radiation, the beamline optics, sample scattering geometry and the X-ray detector will influence the contrast. The optimum signal-to-noise ratio of $g_{2}$ is obtained when the angular source size is the same as the angular acceptance of the detector pixels (Falus et al., 2006). In the present case, the angular source acceptance is collimated to the similar size in both vertical and horizontal directions to obtain the highest $q$ resolution. For partial coherence, the intensity probability distribution function $P(I)$ can be written as

$$
P(I)=I^{M-1}\left(\frac{M}{\langle I\rangle}\right)^{M} \frac{\exp (-M I /\langle I\rangle)}{\Gamma(M)}
$$

(Goodman, 1985), where $M$ is the number of coherence volumes, which is related to the contrast as $\beta=1 / M$.

\subsection{XPCS data}

To demonstrate the XPCS capabilities of the presented setup, we study the free, Brownian diffusion of dilute colloidal particles. The mean-square displacement of the particles $\left\langle\Delta r^{2}\right\rangle$ is related to the free diffusion constant $D_{0}$,

$$
\left\langle\Delta r^{2}\right\rangle=6 D_{0} t .
$$

In this case, the intensity autocorrelation function can be written as a single exponential,

$$
g_{2}(q, t)-1=\beta \exp [-2 \Gamma(q) t] .
$$

Here, $\Gamma(q)$ is the relaxation rate, or $\tau_{c}=1 / \Gamma$ the relaxation time. The factor of 2 arises from the Siegert relation which relates the intensity autocorrelation function to the electric field correlation function (Berne \& Pecora, 2000). For freely diffusing particles, the relaxation rate $\Gamma$ is proportional to the diffusion coefficient,

$$
\Gamma=D_{0} q^{2},
$$

which is given by the Stokes-Einstein relation

$$
D_{0}=\frac{k_{\mathrm{B}} T}{6 \pi \eta R_{\mathrm{H}}} .
$$

where $T, \eta, R_{\mathrm{H}}$ and $k_{\mathrm{B}}$ are the temperature, the viscosity of the suspending medium, the hydrodynamic radius of the particles and the Boltzman constant, respectively.

\section{Experimental}

\subsection{Materials}

Two different samples were used to demonstrate the XPCS capabilities of the presented setup; charge-stabilized silica particles with nominal diameters of 450 and $600 \mathrm{~nm}$, produced via the Stöber synthesis. To increase the concentration of particles, the samples were left to sediment and then the lower part of the solution was used. The samples exhibited strong multiple light scattering, as they appeared milky, demonstrating the difficulty to investigate these samples with conventional DLS. The volume fraction of the $450 \mathrm{~nm}$-sized particle suspension was estimated from the absolute scattering intensity to be 0.01 .

In order to slow down the dynamics and test the presented setup on different timescales, one sample $(600 \mathrm{~nm})$ was transferred into a water/glycerol solution. The water to glycerol ratio was $76.5 \mathrm{wt} \%$. Therefore, the viscosity of the medium is higher by roughly a factor of 41 as compared with pure water, slowing the diffusional motion down correspondingly. The volume fraction was obtained, taking into account the reduced contrast, as 0.004 .

\subsection{Beamline ID02, ESRF}

3.2.1. Source and optics. The X-ray beam is delivered by two phased undulators of period $21.4 \mathrm{~mm}$, length $1.6 \mathrm{~m}$ and minimum gap of about $11 \mathrm{~mm}$. The central cone of the undulator spectrum is selected by primary slits of size $0.2 \mathrm{~mm}$ $\times 0.2 \mathrm{~mm}$. The incident beam is monochromated $(E=$ $12.46 \mathrm{keV})$ by a liquid-nitrogen-cooled channel-cut Si-111 monochromator $\left(\Delta E / E \simeq 2 \times 10^{-4}\right)$. To preserve the source brilliance as much as possible, the focusing optics is based on a high-quality toroidal mirror reflecting in the horizontal plane. This makes the focused beam size less sensitive to longitudinal slope errors and a second horizontally reflecting fine planar mirror maintains the reflected beam parallel to the incident beam (Van Vaerenbergh et al., 2015). Fig. 1 shows the positions of the main optical components. The beam is focused at the detector position about $96 \mathrm{~m}$ from the source.

3.2.2. Detectors. Two different detectors, a Pilatus $300 \mathrm{~K}$ and a FReLoN (Fast-Readout, Low-Noise), were used to record the scattering data. The detectors differ in pixel size, readout time and detection principle. The Pilatus $300 \mathrm{~K}$ is a single-photon-counting hybrid pixel detector and is therefore free of dark current and readout noise. It has an active area of $487 \times 619$ pixels, a pixel size of $172 \mu \mathrm{m} \times 172 \mu \mathrm{m}$ and maximum repetition rate of 400 frames per second. The standard detector for USAXS experiments at ID02 is an integrating FReLoN CCD-based detector, which can be additionally used for XPCS experiments. It features a $20 \mu \mathrm{m}$ thick phosphor screen optically coupled to a Kodak KAF-4320 image sensor $(2048 \times 2048$ pixels $)$ via a fibre-optic plate. It offers smaller effective pixels sizes $(24 \mu \mathrm{m})$ and therefore higher spatial resolution $(\sim 48 \mu \mathrm{m})$ as well as single-photon sensitivity. However, the highest possible sampling rate, in $1 \times 1$ binning, is limited to about $5 \mathrm{~Hz}$. 


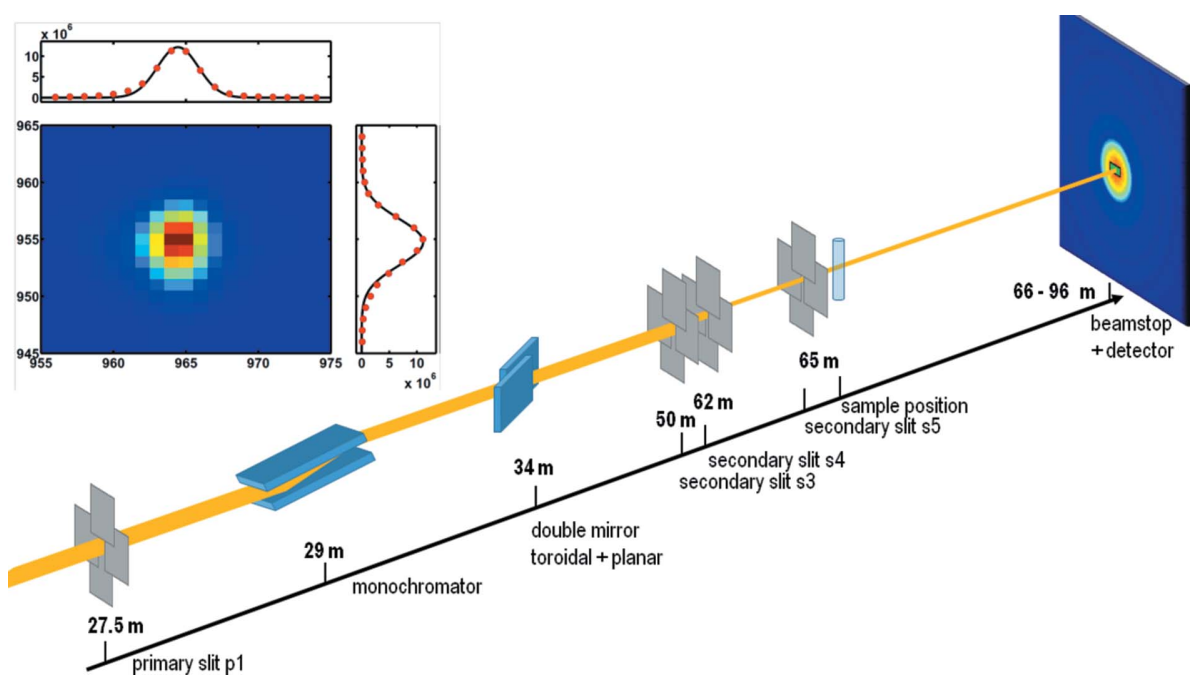

Figure 1

Sketch of the collimation scheme and experimental setup. The inset shows the attenuated direct beam at a distance of $30.7 \mathrm{~m}$ from the sample position, measured with the FReLoN detector.

rate $(0.0002 \mathrm{~s}$ exposure and $0.0023 \mathrm{~s}$ read-out time per image frame).

In addition, the colloidal suspension in the water/glycerol solution was measured with 240 frames at $5 \mathrm{~Hz}$ using the Pilatus and FReLoN detectors, as well as 1024 frames at $200 \mathrm{~Hz}$ using only the Pilatus detector. Exposure times for a single frame were $0.002 \mathrm{~s}(200 \mathrm{~Hz})$ or $0.02 \mathrm{~s}(5 \mathrm{~Hz})$, respectively.

In the case of the FReLoN detector, the measurements were dark image subtracted before further evaluation. As the Pilatus detector has no dark current, this procedure was not required. Both detector images were flatfield corrected and unusable pixels were masked. Data processing was carried out using the PYXPCS software package developed at ID10, ESRF. The $q$ values for the calculation of auto-

3.2.3. Experimental setup. XPCS measurements were performed with an X-ray energy of $12.46 \mathrm{keV}$. A sketch of the experimental setup is shown in Fig. 1. The inset shows the direct beam on the FReLoN detector.

The beam was collimated by a primary slit (p1) in front of the monochromator and two sets of secondary slits $(\mathrm{s} 3, \mathrm{~s} 4)$, which were set to aperture sizes of $40 \mu \mathrm{m} \times 50 \mu \mathrm{m}$ and horizontally shifted by $35 \mu \mathrm{m}$, resulting in an effective slit aperture of $40 \mu \mathrm{m} \times 15 \mu \mathrm{m}$. Furthermore, a guard slit (s5), located $3 \mathrm{~m}$ from the last beam-defining slit, was used to clean parasitic scattering at ultra-low angles. With this arrangement, a well defined and collimated beam could be obtained. The size of the beam on the sample was $40 \mu \mathrm{m} \times 40 \mu \mathrm{m}$. At the detector position it was measured to be $64 \mu \mathrm{m} \times 99 \mu \mathrm{m}$ (see inset of Fig. 1; the FWHM was measured to be $80 \mu \mathrm{m}$ vertical and $110 \mu \mathrm{m}$ horizontal, with the detector resolution of $48 \mu \mathrm{m})$. The resulting flux was $2 \times 10^{11}$ photons s$^{-1}$ and the sample-todetector distance was $30.7 \mathrm{~m}$. Different exchangeable beamstops of various size and shape are installed in the setup. But for XPCS, a circular beamstop of diameter either $1.6 \mathrm{~mm}$ or $3 \mathrm{~mm}$ was used which retained symmetrical azimuthal bins.

The colloidal samples were filled in quartz capillaries of $1 \mathrm{~mm}$ diameter and the temperature was controlled with a Peltier stage. The temperature was kept constant at $20^{\circ} \mathrm{C}$ for the duration of the whole experiment.

\subsection{USAXS-XPCS measurements}

To probe the dynamics of silica particles suspended in pure water, we collected 500 frames using the Pilatus detector operating with a $400 \mathrm{~Hz}$ frame
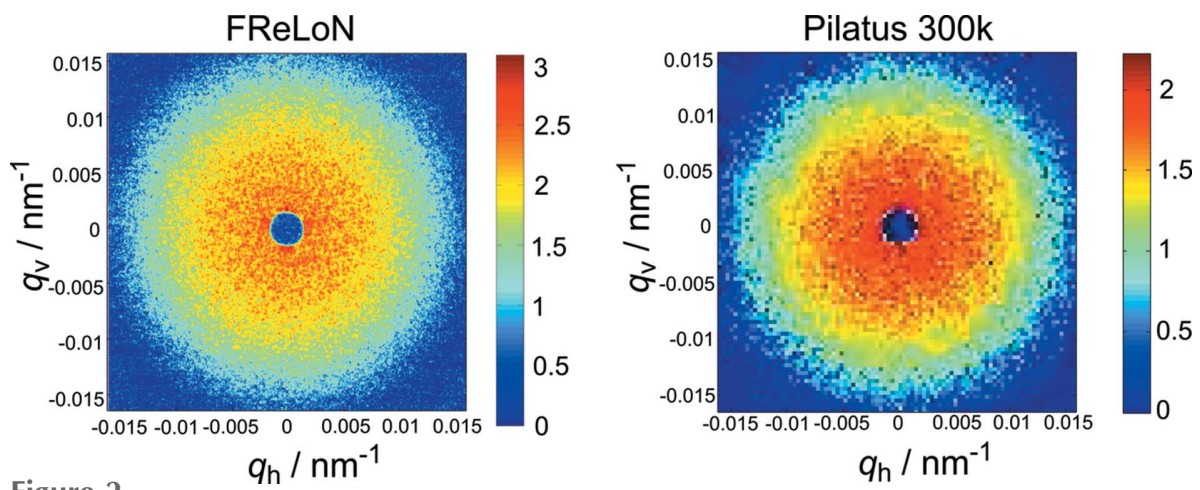

Speckle pattern of the same sample of $450 \mathrm{~nm}$ silica particles in water measured with the FReLoN and Pilatus detectors. The exposure time for both images was $0.2 \mathrm{~ms}$. The beamstop has a diameter of $1.6 \mathrm{~mm}$. 


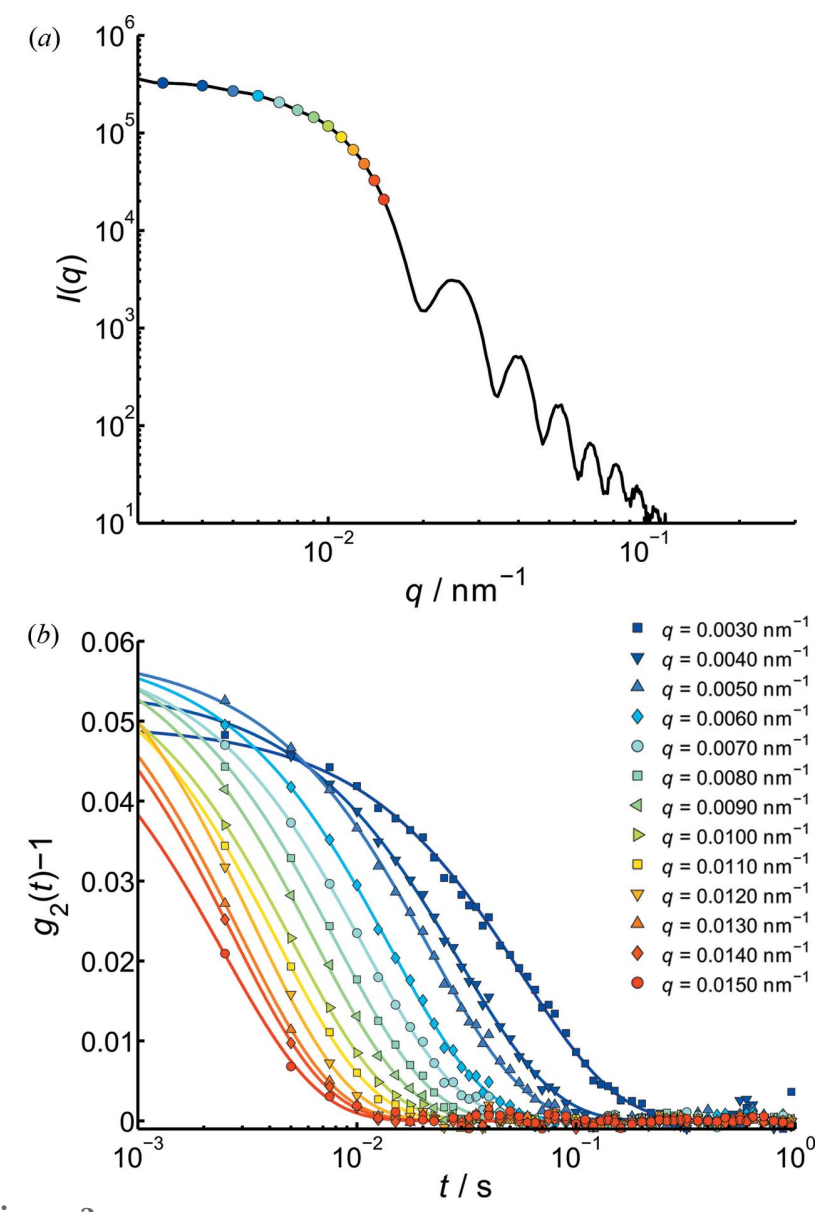

Figure 3

(a) Static scattering intensity and (b) intensity autocorrelation functions of a sample of $450 \mathrm{~nm}$-diameter silica particles in water measured with the Pilatus $300 \mathrm{~K}$ detector. A contrast $\beta=0.05-0.06$ was measured. Additional (coloured) data points in $(a)$ correspond to the $q$-values of the $g_{2}(q, t)$ functions in $(b)$.

was calculated by azimuthally averaging all measured frames. The corresponding autocorrelation functions are displayed in Fig. 3(b). For this sample, the smallest $q$ value at which an autocorrelation function can still be calculated was found to be about $q_{\min }=3 \times 10^{-3} \mathrm{~nm}^{-1}$ with a range of $\Delta q=$ $1 \times 10^{-3} \mathrm{~nm}^{-1}$. Even though the static scattering curve can be measured at smaller values, the corresponding correlation functions could not yield a proper baseline. Note that the determination of the lowest possible $q$ value is not given by a strict criterion, as many different parameters such as the scattering power of the sample in addition to the angular resolution of the detector and size of the beamstop. In general it can be stated that, due to the small number of pixels near to the beamstop, a sufficiently scattering sample is needed in order to calculate successfully the correlation functions at this small $q$ value.

To analyze lower scattering samples at smaller $q$ values, a higher experimental resolution with a larger number of pixels in this area of the detector is needed. This will be further discussed in $\S 4.2$.

The highest $q$ is only given by the maximum acquisition rate of the detector, as the obtained correlation functions are still of good statistics but, as the correlation rate increases with $q^{2}$, they become too fast to be measured.

The plotted autocorrelation functions were fitted using a single exponential function [equation (7)], which presents the measured data very well (solid lines). The obtained contrast was found to be around $\beta=0.05-0.06$. In general, a reduction of the contrast was found for the smallest accessible $q$-values $\left(3 \times 10^{-3}\right.$ to $\left.4 \times 10^{-3} \mathrm{~nm}^{-1}\right)$, which is caused primarily by the reduced number of pixels and residual parasitic background. The possible heterodyne effect due to the higher background scattering near the beamstop is not severe since the obtained $\Gamma(q)$ values are consistent with the expected homodyne decay rates. These decay rates $\Gamma(q)$, given by the exponential fits, are plotted against $q^{2}$ in Fig. 4.

Clearly, a linear relation between $\Gamma$ and $q^{2}$ can be obtained, which arises from the purely diffusive behaviour of the particles dynamics and therefore demonstrates the good performance of the presented setup. Calculating the hydrodynamic radius from the linear refinement using equation (9) gives $R_{\mathrm{H}}=239 \mathrm{~nm}$, which is in reasonable agreement with the value from the static scattering $\left(R_{\text {mean }}=225 \mathrm{~nm}\right)$.

A certain advantage of a two-dimensional detection scheme is the fast acquisition of a large amount of data due to the large number of pixels. This increases not only the statistical quality of the data and considerably decreases the measurement time but allows also a better ensemble averaging of the correlation functions. Furthermore, additional quantities such as the calculation of a two-time correlation function from the measured data can be performed. The two-time correlation function $g_{2}\left(t_{1}, t_{2}\right)$ of the previous displayed data is calculated for a scattering vector $q=4 \times 10^{-3} \mathrm{~nm}^{-1}$ and shown in Fig. 5 . As expected for a purely diffusive system, no time evolution (aging) can be seen in these data. Furthermore, it shows that no radiation damage is present or the sample is not evolving during the measurement.

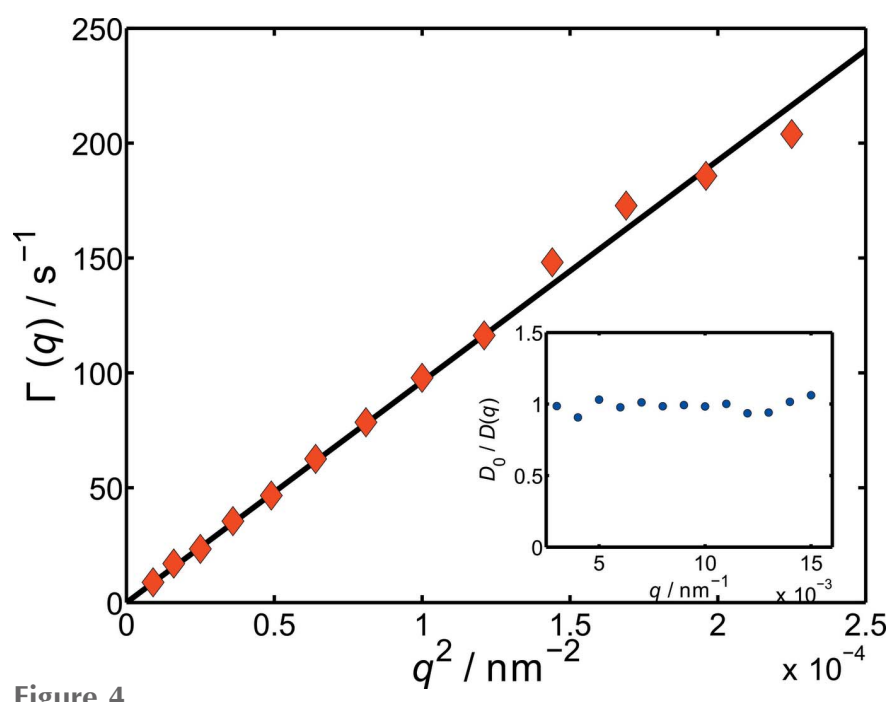

Figure 4

Results of the fits with equation (7) to the data in Fig. 3. A linear relation between $\Gamma$ and $q^{2}$ can be seen, corresponding to purely diffusive particle dynamics. Inset: $D_{0} / D(q)$ plotted versus $q . D(q)$ is the measured diffusion coefficient at each $q$, with $D(q)=\Gamma(q) / q^{2}$. 


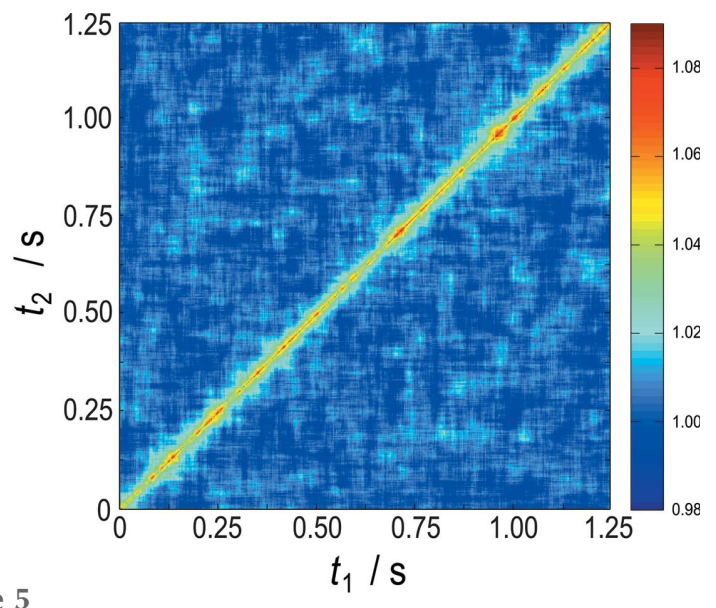

Figure 5

Two-time correlation function $g_{2}\left(t_{1}, t_{2}\right)$ at $q_{\min }=4 \times 10^{-3} \mathrm{~nm}^{-1}$.

\subsection{Comparison between different detection schemes}

In order to investigate the influence of the detector characteristics on the quality of the obtained XPCS data, measurements were additionally performed using the Pilatus and the FReLoN detectors. The silica particles were suspended in a glycerol/water solution to slow down the dynamics of the colloidal system. The obtained correlation functions are displayed in Figs. 6 and 7.

The two detectors differ in two principle characteristics. The Pilatus is a fast detector $(400 \mathrm{~Hz})$, allowing access to smaller correlation times $t$, but has rather large pixels. The FReLoN is slower $(5 \mathrm{~Hz})$, but it has seven times smaller pixels. This smaller pixel size of the FReLoN detector has two impacts on the obtained data. In general, a smaller pixel size can be translated as a higher angular resolution of the experiment. In the case of the Pilatus $300 \mathrm{~K}$, each speckle is within one pixel, while the same speckle distributes over a cluster of pixels on the FReLoN detector. This should result in a higher contrast $\beta$. That this is the case can directly be demonstrated with the

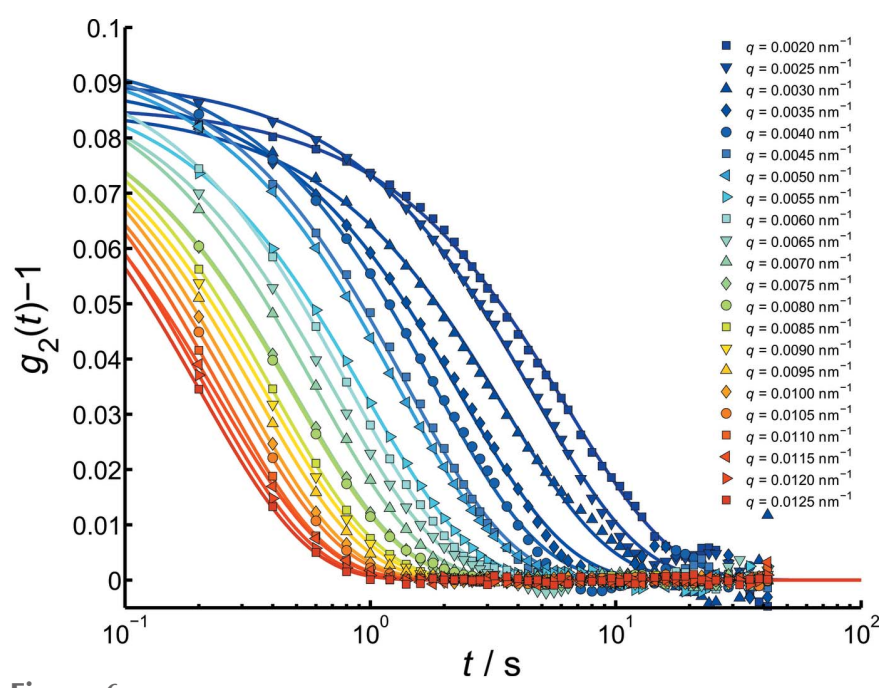

Figure 6

Intensity autocorrelation function of $600 \mathrm{~nm}$ silica particles in water/ glycerol measured with the FReLoN detector at $5 \mathrm{~Hz}$.

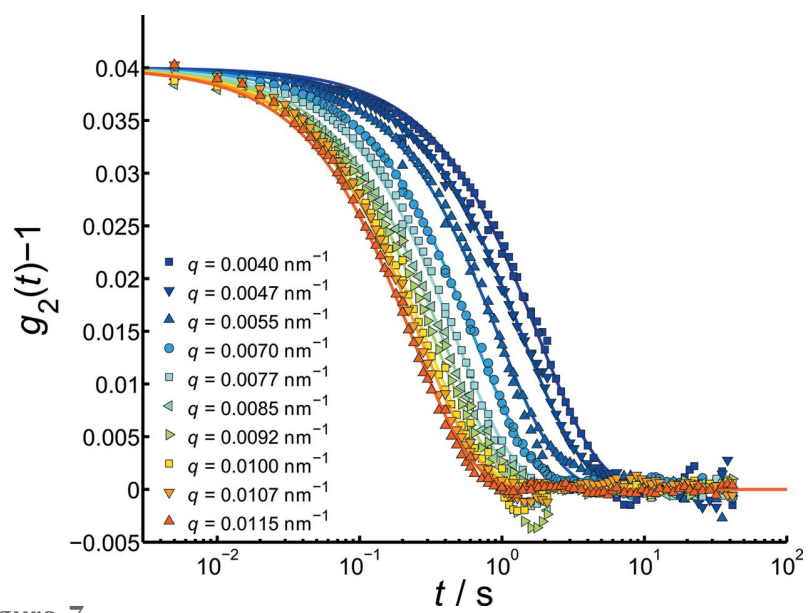

Figure 7

Intensity autocorrelation function of $600 \mathrm{~nm}$ silica particles in water/ glycerol measured with the Pilatus detector at $200 \mathrm{~Hz}$ and $5 \mathrm{~Hz}$.

contrast values obtained from the $g_{2}(t)$ data. The contrast obtained by using the Pilatus detector is $\beta \cong 0.04$, whereas the higher angular resolution of the FReLoN detector gives a contrast of $\beta \cong 0.08-0.09$.

Furthermore, a much smaller $q_{\text {min }}$ can be chosen in the data treatment process as more pixels near to the beamstop contribute to the signal $\left(q_{\min }=2 \times 10^{-3} \mathrm{~nm}^{-1}\right.$ for the FReLoN and $q_{\text {min }}=3 \times 10^{-3} \mathrm{~nm}^{-1}$ for the Pilatus detector $)$. As the correlation rate is proportional to $q^{2}$, these correlation functions exhibit also much slower decay rates. Furthermore, due to the smaller pixel size and therefore larger number of pixels, a smaller sectioning (FReLoN: $\Delta q_{i}=0.4 \times 10^{-3} \mathrm{~nm}^{-1}$; Pilatus: $\left.\Delta q_{i}=0.75 \times 10^{-3} \mathrm{~nm}^{-1}\right)$ can be chosen.

The resulting correlation rates are displayed in Fig. 8. The data points are fitted and the hydrodynamic radius obtained was found to be $R_{\mathrm{H}}=316 \mathrm{~nm}$. No quantitative difference between the data obtained with the two different detectors can be observed. It clearly shows, however, the advantage of a higher angular resolution of the experimental setup, as the

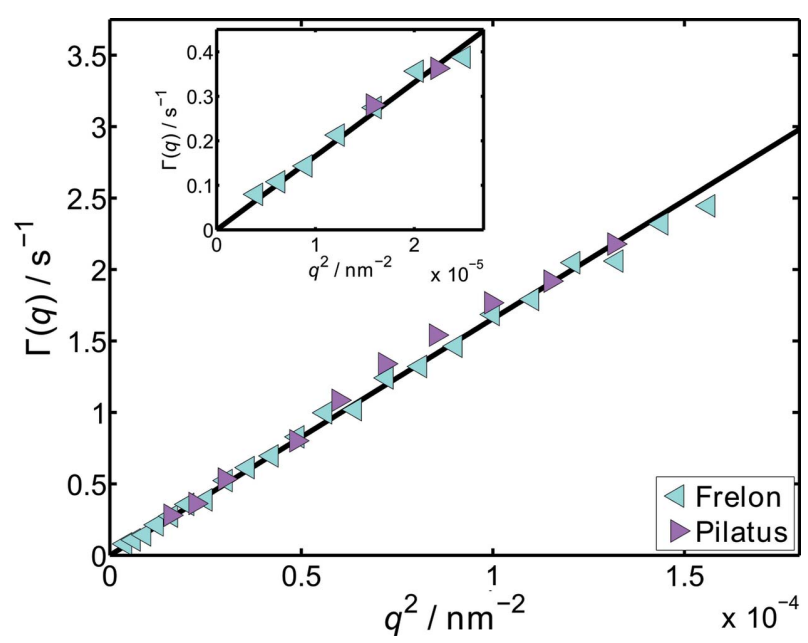

Figure 8

Decay rates of the $600 \mathrm{~nm}$ silica particles in a glycerol/water solution obtained with the two different detectors. The inset shows a zoom into the low $q$ region. 


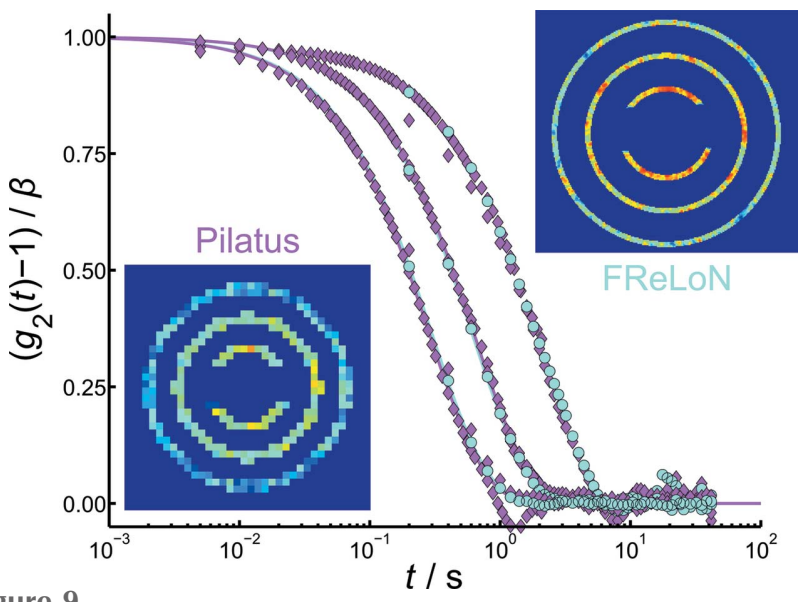

Figure 9

Intensity autocorrelation function obtained with the Pilatus $300 \mathrm{~K}$ and FReLoN detector at $q=4 \times 10^{-3} \mathrm{~nm}^{-1}, q=7 \times 10^{-3} \mathrm{~nm}^{-1}$ and $q=$ $1 \times 10^{-2} \mathrm{~nm}^{-1}$. The insets show the corresponding two-dimensional scattering patterns.

correlation rates can be determined on a larger $q$ range and with a smaller step size. Especially when accessing the hydrodynamic structure factor from the XPCS data, this feature may be an advantage. It can be stated that a higher angular resolution, even though with limited temporal resolution, might give data of higher quality.

In the following, the data given by the two detectors are further compared. Fig. 9 shows three representative correlation functions at $q=4 \times 10^{-3} \mathrm{~nm}^{-1}, q=7 \times 10^{-3} \mathrm{~nm}^{-1}$ and $q=1 \times 10^{-2} \mathrm{~nm}^{-1}$ each measured with the two detectors. The insets show the corresponding detector image, where only the pixels corresponding to the three $q$ ranges are not masked. The higher number of pixels corresponding to the signal of each $q$ range in the case of the FReLoN detector is obvious. The sample-to-detector distance is the same for both detectors.

According to equation (4), the probability distribution of intensity $P(I)$ in one of the displayed rings should follow an exponential decay in the case of a fully coherent experiment, i.e. $\beta=1$. For experiments with lower coherence $\left(\beta_{\text {FReLoN }} \simeq\right.$ 0.09 and $\beta_{\text {Pilatus }} \simeq 0.04$ as obtained from the correlation functions), the distribution of intensities should follow equation (5).

In Fig. 10, the intensity distributions measured with the two detectors are shown for $q=4 \times 10^{-3} \mathrm{~nm}^{-1}, q=7 \times 10^{-3} \mathrm{~nm}^{-1}$ and $q=1 \times 10^{-2} \mathrm{~nm}^{-1}$. The distributions were calculated from each two-dimensional image separately and then the distributions from all frames were averaged to achieve better statistics [FReLoN: 240 frames; Pilatus: 240 frames $(5 \mathrm{~Hz})+$ 1024 frames $(200 \mathrm{~Hz})]$. The resulting distributions were fitted using equation (5), with $M=1 / \beta$ being the only free parameter (dashed line). Only a moderate agreement with the data can be obtained. The agreement can be improved, however, if a constant value $(1-\alpha)$ is subtracted from the scaled intensity before the mean value is calculated (continuous line). This procedure corresponds to the assumption that a constant part of the incident beam does not contribute to the coherent interference which results in the speckle pattern (Abernathy et
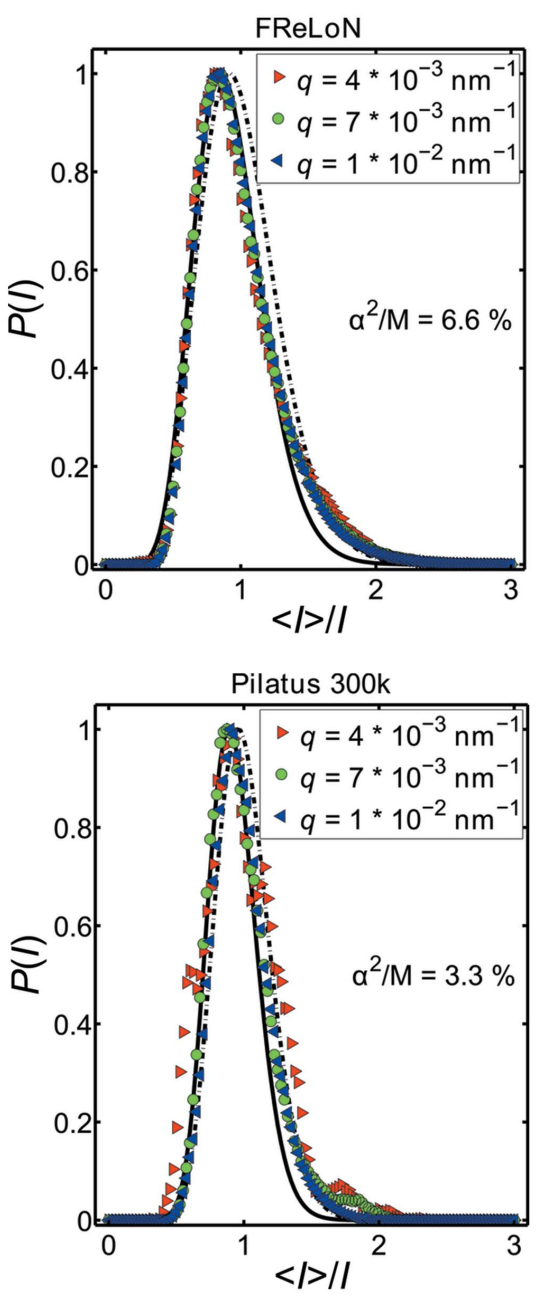

Figure 10

Intensity distributions $P(I)$ at $q=4 \times 10^{-3} \mathrm{~nm}^{-1}, q=7 \times 10^{-3} \mathrm{~nm}^{-1}$ and $q=1 \times 10^{-2} \mathrm{~nm}^{-1}$ measured with the FReLoN and Pilatus detectors, respectively.

al., 1998; Grübel et al., 2008). In our case, we found that a value of $\alpha=0.925$ is needed to sufficiently describe the data. The contrast is calculated as $\beta=\alpha^{2} / M$ (Grübel et al., 2008), resulting in $\beta_{\text {FReLoN }}=0.066$ and $\beta_{\text {Pilatus }}=0.033$. In both cases, slightly smaller values than the previously obtained contrasts from refining the correlation functions are found. However, the roughly doubled contrast of the FReLoN detector is evident. Furthermore, the intensity distributions are not significantly modified and follow the prediction even though fundamentally different detection principles are involved. It can be stated that an integrating CCD-type detector having single-photon sensitivity can be used for XPCS experiments and both detectors show similar statistical properties. Due to the reduced pixel size and therefore higher angular resolution, the FReLoN detector even provides better results for samples with slower dynamics and non-equilibrium systems.

\section{Concluding remarks}

We have demonstrated the feasibility of performing highquality XPCS measurements in the USAXS range using a 
high-resolution pinhole collimation instrument with $1 \AA \mathrm{X}$-ray wavelength. The correlation functions can be obtained with high resolution in much shorter measurement times as compared with previous setups using a Bonse-Hart USAXS camera. The presented setup offers several new possibilities, like studying non-equilibrium systems and obtaining two-time correlation functions over the scattering vector range comparable with small-angle light scattering. We would like to point out that several experimental applications may become accessible with this new setup, which were out of the feasible range of DLS and XPCS. Examples include the dynamics of self-propelled colloids, local velocity fluctuations in concentrated sedimenting suspensions, collective movement in ageing colloidal gels, dynamics of drying colloidal film such as paint, and so on.

Furthermore, it has been demonstrated that two detectors with different X-ray detection principles can successfully be used to measure XPCS data. The high time resolution of the Pilatus and the high angular resolution of the FReLoN detector are complementary for different kinds of samples. The obtained data were found to be of similar statistical quality. A further improvement in the future may be achieved with more advanced detectors, combining high time and high spatial resolution, like for example with the Eiger pixel detector.

\section{Acknowledgements}

The assistance and help of Gudrun Lotze, Enrico Semeraro, Michael Sztucki, Rajeev Dattani and Jacques Gorini are kindly acknowledged. ESRF is thanked for providing synchrotron beam time.

\section{References}

Aberle, L. B., Hülstede, P., Wiegand, S., Schröer, W. \& Staude, W. (1998). Appl. Opt. 37, 6511-6524.

Abernathy, D. L., Grübel, G., Brauer, S., McNulty, I., Stephenson, G. B., Mochrie, S. G. J., Sandy, A. R., Mulders, N. \& Sutton, M. (1998). J. Synchrotron Rad. 5, 37-47.

Alaimo, M. D., Potenza, M. A. C., Manfredda, M., Geloni, G., Sztucki, M., Narayanan, T. \& Giglio, M. (2009). Phys. Rev. Lett. 103, 194805.

Berne, B. J. \& Pecora, R. (2000). Dynamic Light Scattering: With Applications to Chemistry, Biology, and Physics. New York: Dover. Block, I. D. \& Scheffold, F. (2010). Rev. Sci. Instrum. 81, 123107.

Brown, G., Rikvold, R. A., Sutton, M. \& Grant, M. (1997). Phys. Rev. $E$, 56, 6601-6612.

Burghardt, W. R., Sikorski, M., Sandy, A. R. \& Narayanan, S. (2012). Phys. Rev. E, 85, 021402.
Cerbino, R., Peverini, L., Potenza, M. A. C., Robert, A., Bösecke, P. \& Giglio, M. (2008). Nat. Phys. 4, 238-243.

Falus, P., Lurio, L. B. \& Mochrie, S. G. J. (2006). J. Synchrotron Rad. 13, 253-259.

Fluerasu, A., Kwasniewski, P., Caronna, C., Destremaut, F., Salmon, J.-B. \& Madsen, A. (2010). New J. Phys. 12, 035023.

Fluerasu, A., Moussaïd, A., Madsen, A. \& Schofield, A. (2007). Phys. Rev. E, 76, 010401.

Goodman, J. (1985). Statistical Optics. New York: Wiley.

Grübel, G., Madsen, A. \& Robert, A. (2008). Soft Matter Characterization, edited by R. Borsali and R. Pecora, ch. 18, pp. 954-995. Berlin/Heidelberg: Springer.

Hoshino, T., Kikuchi, M., Murakami, D., Harada, Y., Mitamura, K., Ito, K., Tanaka, Y., Sasaki, S., Takata, M., Jinnai, H. \& Takahara, A. (2012). J. Synchrotron Rad. 19, 988-993.

Jakeman, E., Pusey, P. N. \& Vaughan, J. M. (1976). Opt. Commun. 17, 305-308.

Llopart, X., Campbell, M., Dinapoli, R., San Segundo, D. \& Pernigotti, E. (2002). IEEE Trans. Nucl. Sci. 49, 2279-2283.

Lu, X., Mochrie, S. G. J., Narayanan, S., Sandy, A. R. \& Sprung, M. (2011). J. Synchrotron Rad. 18, 823-834.

Madsen, A., Leheny, R. L., Guo, H., Sprung, M. \& Czakkel, O. (2010). New J. Phys. 12, 055001.

Mainville, J., Bley, F., Livet, F., Geissler, E., Legrand, J. F., Abernathy, D., Grübel, G., Mochrie, S. G. J. \& Sutton, M. (1997). J. Appl. Cryst. 30, 828-832.

Moussaïd, A. \& Pusey, P. N. (1999). Phys. Rev. E, 60, 5670-5676.

Pine, D. J., Weitz, D. A., Chaikin, P. M. \& Herbolzheimer, E. (1988). Phys. Rev. Lett. 60, 1134-1137.

Sandy, A. R., Lurio, L. B., Mochrie, S. G. J., Malik, A., Stephenson, G. B., Pelletier, J. F. \& Sutton, M. (1999). J. Synchrotron Rad. 6, 1174-1184.

Segrè, P. N., Behrend, O. P. \& Pusey, P. N. (1995a). Phys. Rev. E, 52, 5070-5083.

Segrè, P. N., Van Megen, W., Pusey, P. N., Schätzel, K. \& Peters, W. (1995b). J. Mod. Opt. 42, 1929-1952.

Shinohara, Y., Imai, R., Kishimoto, H., Yagi, N. \& Amemiya, Y. (2010a). J. Synchrotron Rad. 17, 737-742.

Shinohara, Y., Kishimoto, H., Yagi, N. \& Amemiya, Y. (2010b). Macromolecules, 43, 9480-9487.

Sutton, M. (2008). C. R. Phys. 9, 657-667.

Sztucki, M. \& Narayanan, T. (2007). J. Appl. Cryst. 40, s459-s462.

Sztucki, M., Narayanan, T., Belina, G., Moussaïd, A., Pignon, F. \& Hoekstra, H. (2006). Phys. Rev. E, 74, 051504.

Van Vaerenbergh, P., Leonardon, J., Sztucki, M., Bösecke, P., Gorini, J., Claustre, J., Sever, F., Morse, J. \& Narayanan, T. (2015). 12th International Conference on Synchrotron Radiation Instrumentation (SRI-2015), 6-10 July 2015, New York, USA.

Westermeier, F., Autenrieth, T., Gutt, C., Leupold, O., Duri, A., Menzel, A., Johnson, I., Broennimann, C. \& Grübel, G. (2009). J. Synchrotron Rad. 16, 687-689.

Zhang, F., Allen, A. J., Levine, L. E., Ilavsky, J. \& Long, G. G. (2012). Met. Mater. Trans. A, 43, 1445-1453.

Zhang, F., Allen, A. J., Levine, L. E., Ilavsky, J., Long, G. G. \& Sandy, A. R. (2011). J. Appl. Cryst. 44, 200-212. 